\title{
Study of the Dependency on Magnetic Field and Bias Voltage of an AC-Biased TES Microcalorimeter
}

\author{
L. Gottardi · J. Adams · C. Bailey • S. Bandler • M. Bruijn · J. Chervenak • \\ M. Eckart • F. Finkbeiner • R. den Hartog • H. Hoevers • R. Kelley • \\ C. Kilbourne · P. de Korte · J. van der Kuur • M. Lindeman • F. Porter • \\ J. Sadlier • S. Smith
}

Received: 3 August 2011 / Accepted: 4 January 2012 / Published online: 21 January 2012

(C) The Author(s) 2012. This article is published with open access at Springerlink.com

\begin{abstract}
At SRON we are studying the performance of a Goddard Space Flight Center single pixel TES microcalorimeter operated in an AC bias configuration. For X-ray photons at $6 \mathrm{keV}$ the pixel shows an X-ray energy resolution $\Delta E_{F W H M}=3.7 \mathrm{eV}$, which is about a factor 2 worse than the energy resolution observed in an identical DC-biased pixel. In order to better understand the reasons for this discrepancy we characterised the detector as a function of temperature, bias working point and applied perpendicular magnetic field. A strong periodic dependency of the detector noise on the TES AC bias voltage is measured. We discuss the results in the framework of the recently observed weak-link behaviour of a TES microcalorimeter.
\end{abstract}

Keywords x-ray detector $\cdot$ SQUID $\cdot$ rf-SQUID $\cdot$ TES $\cdot$ LC resonator

\section{Introduction}

In previous works we compared the performances of an X-ray TES microcalorimeter under AC and DC bias by measuring the IV characteristic, the noise, the impedance and the X-ray response at perpendicular magnetic field $B_{\perp}=0$. The tests were carried out both with an SRON pixel and a GSFC pixel [1,2]. With respect to the DC bias case, under AC bias we observe a smaller (about 15\%) current output at small voltage bias low in the transition, a low TES current and temperature sensitivity, a slightly worse integrated NEP resolution and about a factor two $\mathrm{x}$-ray resolution degradation.

L. Gottardi $(\varangle) \cdot$ M. Bruijn · R. den Hartog $\cdot$ H. Hoevers · P. de Korte · J. van der Kuur · M. Lindeman

SRON National Institute for Space Research, Sorbonnelaan 2, 3584 CA Utrecht, The Netherlands e-mail: 1.gottardi@sron.nl

J. Adams · C. Bailey · S. Bandler · J. Chervenak · M. Eckart · F. Finkbeiner · R. Kelley ·

C. Kilbourne $\cdot$ F. Porter $\cdot$ J. Sadlier $\cdot$ S. Smith

NASA GSFC, Greenbelt Road, Greenbelt, MD 20771, USA 


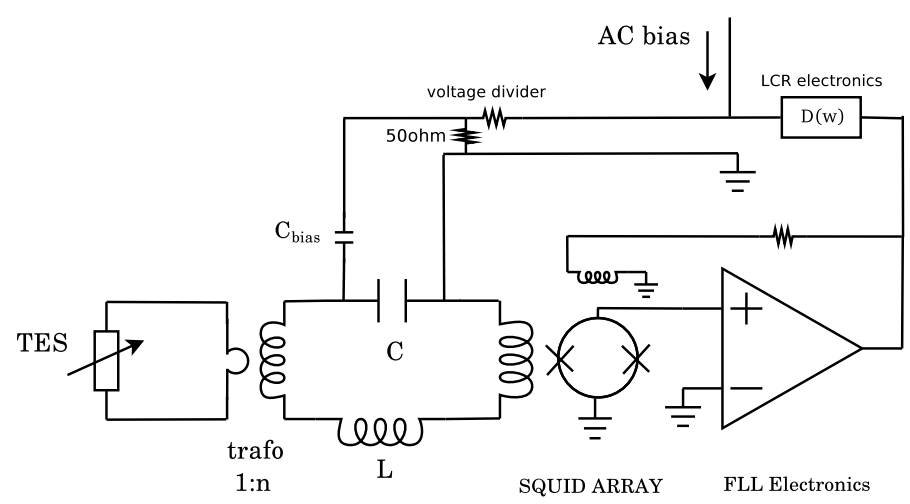

Fig. 1 Schematic drawing of the AC bias and read-out circuit used for the TES microcalorimeter. A superconducting flux transformer is used to improve the impedance matching between the SQUID amplifier and the TES microcalorimeter

In order to better understand the reasons for the suboptimal performance of the TESs under AC bias we thoroughly studied the effect of the perpendicular magnetic field and the voltage bias on the detector response. Here we present the results obtained with a GSFC pixel read out both in the AC and DC configuration.

\section{Experimental Details}

A schematic drawings of the read-out circuit used for the AC measurements described here is shown in Fig. 1. A superconducting flux transformer is used between the SQUID amplifier and the TES microcalorimeter to improve the impedance matching between the TES and SQUID amplifier.

The TES microcalorimeter is tested using a superconducting transformer with inductances $L_{p}=100 \mathrm{nH}$ and $L_{s}=6.4 \mu \mathrm{H}$, and estimated mutual inductance $M=k^{2} L_{s} N_{p} / N_{s}=760 \mathrm{nH}, N_{r}=N_{s} / N_{p}=8$ coil turns ratio and with $L_{p}$ connected to the TES. The impedance of the LC circuit seen by the TES is then $Z_{L C \text {, tes }}=Z_{L C} / N_{r}^{2}$. The LC resonator consists of an hybrid filter with a lithographic Nb-film coil with $L<10 \mathrm{nH}$ and commercial high-Q NP0 SMD capacitors with $C=100 \pm 10 \mathrm{nF}$. The circuit has an additive total stray inductance of about $L_{\text {stray }} \sim 200 \mathrm{nH}$. The intrinsic resonator factor of the LC resonator is $350 \pm 20$, limited by losses in the lumped elements circuit.

As amplifiers we used a NIST SQUID arrays consisting of a series of $100 \mathrm{dc}$ SQUID with input-feedback coil turns ratio of 3:1, and input inductance $L_{\text {in }}=70 \mathrm{nH}$. The input current noise is $\sim 4 \mathrm{pA} / \sqrt{\mathrm{Hz}}$ at $T<1 \mathrm{~K}$. The SQUID amplifier is operated in a standard analogue flux-locked-loop (FLL) mode using commercial Magnicon electronics, which linearises the SQUID response. Only at frequencies below $f<700 \mathrm{kHz}$ the FLL has enough loop gain to sufficiently linearise the signal response. For this reason our experiments are carried out at a resonant frequency of $470 \mathrm{kHz}$.

The circuit resonant frequency is defined by the capacitor $\mathrm{C}$ and the total inductance $L_{t o t}=L_{i n}+L+L_{s t r a y}+L_{f b}+L_{s}-M_{p s}^{2} /\left(L_{p}\right)$, where the last term accounts 

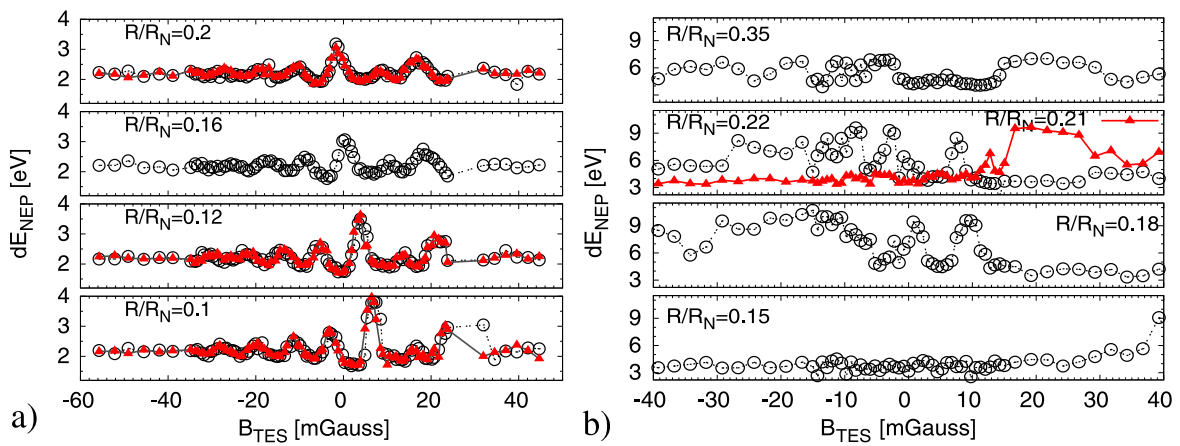

Fig. 2 (Color online) Integrated NEP as a function of magnetic field and for several bias point with TES DC biased (a) and AC biased (b). The cancelling magnetic field for the DC and AC bias pixels are respectively -228 and 68 mGauss. The points in red up triangles are the results of measurements taken under identical experimental condition, but on a different day

for the screening effect of the superconducting transformer and $L_{f b}$ is an inductance generated by the SQUID feedback loop. From the measured resonant frequency and the filter capacitance reported above we get $L_{t o t}=1.1 \mu \mathrm{H}$. The effective inductance seen by the TES is $L_{\text {eff }, T E S}=1.1 \mu \mathrm{H} / N_{r}{ }^{2}=17 \mathrm{nH}$.

In the experiment described here we used an xray TES microcalorimeter from the GSFC. It is a $150 \times 150 \mu \mathrm{m}^{2}$ pixel from a uniform $8 \times 8$ array [3, 4], where a TES is coupled to a micron-thick overhanging $\mathrm{Au} / \mathrm{Bi} \mathrm{X}$-ray absorber. The sensor is a $\mathrm{Mo} / \mathrm{Au}$ proximity-effect bilayer with a transition temperature of $T_{C}=95 \mathrm{mK}$, and a normal state resistance of $R_{N}=7 \mathrm{~m} \Omega$.

\section{Experimental Results}

When the GSFC pixel is DC biased good baseline and x-ray energy resolution is observed. The x-ray resolution is comparable to the baseline resolution and is generally of the order of 2.3-2.5 eV. The pixel responsivity and noise strongly depends on the perpendicular magnetic field applied to the TES. In Fig. 2a. we show the integrate NEP $\left(d E_{N E P}\right)$ as a function of the applied magnetic field $B$ and for different bias current. At this pixel we observed a remanent perpendicular field of $B=228$ mGauss. The pattern observed is due to the dependency of the detector critical current on the magnetic field as a result of the TES behaving as a weak-link [5]. The shift of the Josephson patterns along the applied magnetic field is due to the self magnetic field generated by the DC current flowing through the leads connecting the TES [12].

Under AC bias we measured the $d E_{N E P}$ as a function of the applied perpendicular magnetic field $B$ as well. At this pixel we observed a remanent perpendicular field of $B=-68$ mGauss. The results are shown in Fig. $2 \mathrm{~b}$.

From the plots we observe that, under AC bias: the Fraunhofer-like oscillations are visible, but the pattern is more noisy and less reproducible than under DC bias; the baseline resolution is slightly worse and strongly depends on the bias voltage. While the former effect is likely due to the self magnetic field, the latter has a less 

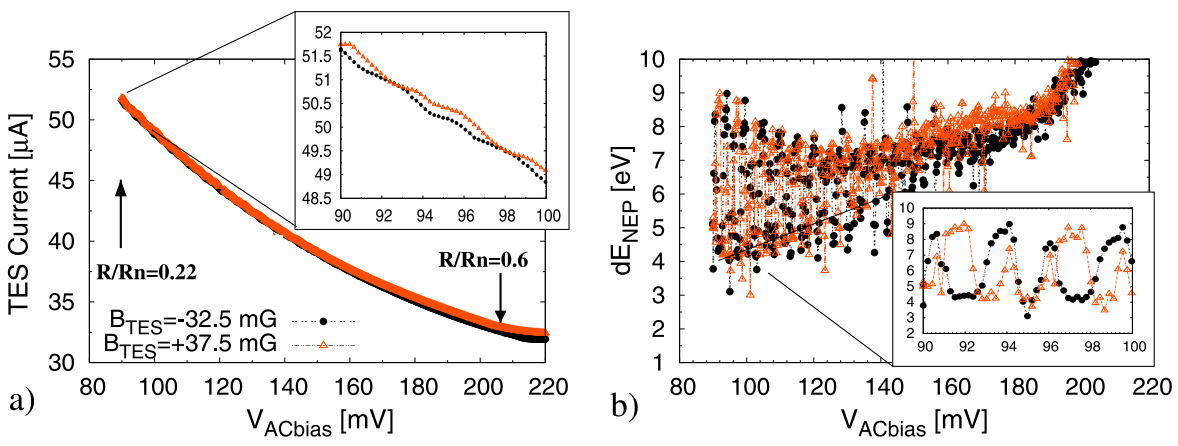

Fig. 3 (Color online) Signal amplitude (i.e. $I_{r m s, T E S}$ TES current) and integrated energy resolution as a function of the bias voltage of the AC biased pixel. The energy resolution shows an oscillating pattern as a function of the bias point. The pattern is partially modulated by the magnetic field. $T_{\text {bath }}=65 \mathrm{mK}$

Fig. 4 Integrated energy resolution as a function of the bias voltage. The energy resolution deteriorates at the bias point corresponding to the higher slope in the IV curve steps. $T_{\text {bath }}=18 \mathrm{mK}$

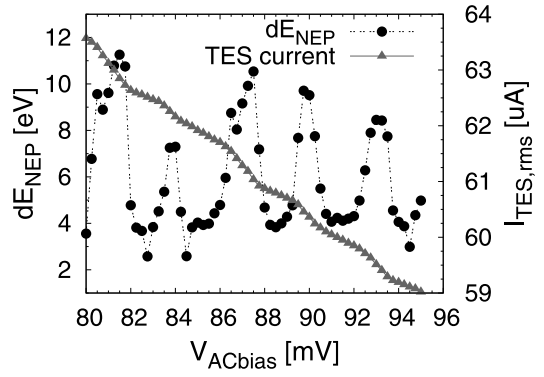

trivial explanation. In an attempt to clarify these results, we performed a fine scan of the detector $\mathrm{I}-\mathrm{V}$ characteristic by measuring for every bias point the detector $\mathrm{x}$-ray response and the noise. We repeated the scan for different magnetic fields. We discovered that the baseline energy resolution strongly depends on the detector bias point. A very small variation $(\sim 2 \%)$ of the bias current could result in an energy resolution degradation of more than a factor three.

The results of this measurement are shown in Fig. 3, where the baseline resolution is plotted as a function of the bias voltage and for several value of the perpendicular magnetic field.

The energy resolution oscillates between values from $3.5 \mathrm{eV}$ to $9 \mathrm{eV}$ as a function of the bias point. The oscillating pattern is partially modulated by the magnetic field.

As visible in the insert of Fig. 3a, the sensor IV curve presents a staircase structure, modulated by the perpendicular magnetic field.

The worst resolution is measured at the transition between two steps where the slope of the I-V curve is the highest. This is clearly seen in Fig. 4.

In Fig. 5 we plot the NEP, the responsivity and the noise spectra taken under AC and DC bias. For the AC bias case the spectra are taken respectively at the flat and at the rising part of the observed steps in Fig. 4. The detector response bandwidth is not identical under AC and DC bias due to the different load impedance of the two circuits $[1,2]$. One should refer to the NEP plot when comparing the AC and DC cases. The NEP is the lowest under DC bias. At low frequency $(f<100 \mathrm{~Hz})$ the 

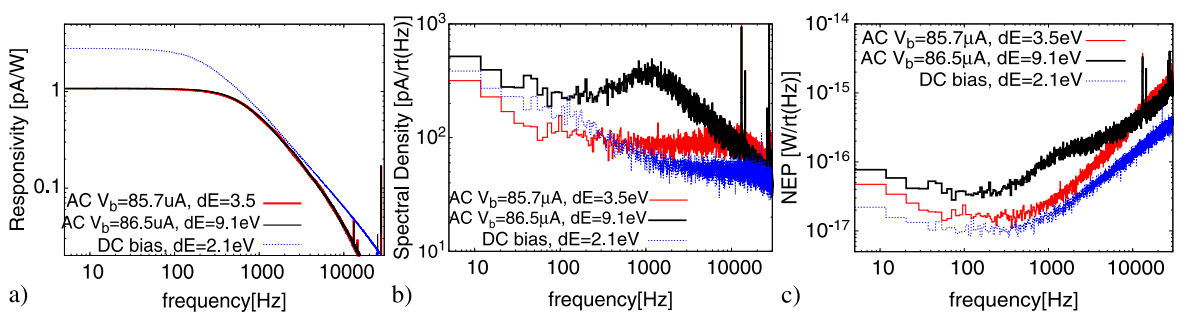

Fig. 5 (Color online) Responsivity (a), noise (b) and NEP (c) measured under DC bias and AC bias. For the $\mathrm{AC}$ bias case the responsivity for two bias points is shown, taken respectively at the flat (red curve) and at the rising (black curve) part of the step

degradation in the NEP observed in the AC bias case is probably due to a reduced responsivity. At high frequency $(f>1 \mathrm{kHz})$ excess noise is observed in the AC bias case. The excess noise is worse for the measurement taken at the rising part of the steps and has the signature of excess Johnson noise. Furthermore, the noise level at frequency $f>1 \mathrm{kHz}$ in the spectra corresponding to the $3.5 \mathrm{eV}$ integrated NEP taken under AC bias, cannot be explained by simply including the SQUID and the $\mathrm{LC}$ resonator thermal noise. An excess white noise of about $50 \mathrm{pA} / \sqrt{\mathrm{Hz}}$ is estimated from the model. Under AC bias the responsivity is independent on the two bias points.

\section{Discussion}

In analogy with the analysis done for an rf-SQUID [6-11] we calculate the characteristic parameters of a TES in a superconducting loop weakly coupled via a superconducting transformer to an LC resonant circuit. The TES is treated as a weak-link in accordance with the RSJ model where $R_{\text {shunt }}$ is assumed to be TES normal resistance. For the detector described above we find the cut-off and the characteristic frequency to be respectively $\omega_{\text {cut }}=R / L \sim 400 \mathrm{kHz}, \omega_{J J}=2 \pi R I_{c} / \Phi_{0} \sim 100 \mathrm{MHz}$, where a critical current of about $I_{c} \sim 5 \mu \mathrm{A}$ is assumed. Note that the TES critical current at a $T_{\text {bath }} \ll T_{c}$ is generally larger than $5 \mu \mathrm{A}(700 \mu \mathrm{A}$ at $55 \mathrm{mK}$ [12]). However in bias conditions the TES operating temperature is $T \sim T_{c}$ and the critical current drops. The screening parameter is $\beta_{r f}=2 \pi L I_{0} / \Phi_{0} \sim 273$. The rfSQUID-like AC biased TES operates then in an adiabatic and hysteretic regime, since $\omega_{L C}<\min \left(\omega_{J J}, \omega_{c u t}\right)$ and $\beta_{r f} \gg 1$.rf-SQUIDs have shown the highest noise in this working regime [11].

The steps in the TES IV curves have a non zero slope (Fig. 3a). The tilting of the steps in the rf-SQUID I-V characteristic and the rounding of the step edges reflect directly the width of the quantum transitions distribution due to thermal fluctuations. Kurkijärvi $[7,8]$ have shown that the ratio $\alpha$ of the voltage rise along a step $\Delta V_{s}$ to the voltage difference $\Delta V_{0}$ between steps is directly proportional to the SQUID's intrinsic flux noise [6]. Their empirical formula gives $\alpha=\frac{1}{0.7 \Phi_{0}}\left(\frac{\omega_{r f}}{2 \pi}\right)^{1 / 2} \sqrt{S_{\Phi}}$. In the same way we can estimate the flux noise in the TES superconducting ring. From the step observed in the IV curves we get an $\alpha=\Delta V_{s} / \Delta V_{0}=0.36$, which corresponds to a flux noise of $\sqrt{S_{\Phi}} \sim 4 \cdot 10^{-4} \frac{\Phi_{0}}{\sqrt{\mathrm{Hz}}}$. For a $17 \mathrm{nH}$ inductance ring this is equivalent to a 
current noise of $\sqrt{S_{I}} \sim 5 \cdot 10^{-11} \frac{\mathrm{A}}{\sqrt{\mathrm{Hz}}}$. This noise level is comparable with the excess noise observed in the AC bias detector, which limits the best measured integrated NEP to $3.5 \mathrm{eV}$.

\section{Conclusions}

We observed excess noise and low reproducibility in the AC biased x-ray pixel. A strong dependency of the baseline noise on the bias voltage is at the origin of the non reproducible results obtained in the past. In the IV curve under AC bias a staircase structure is observed. A possible interpretation of this effect is given by comparing the detector and the AC read-out to an rf-SQUID. In this analogy the TES is seen as a weak-link in a superconducting ring weakly coupled to an LC resonator. Should this interpretation be valid, the excess noise observed in the $\mathrm{AC}$ bias experiment could be due to flux noise generated by the uncertainties in the quantum transition.

To validate this hypothesis the following tests should be performed in the future: measurements with large $L$ and high bias frequency $(f>1 \mathrm{MHz})$ since in the rfSQUID the flux noise decreases at higher rf tank frequencies and the detector operates in a non-adiabatic regime where rf-SQUID amplifiers show lower flux noise [11]; measurements of excess noise (integrated NEP) as function of temperature since a weak dependency to $T^{2 / 3}$ is expected [7,8]; experiments with TESs with different weak-link parameters $\left(I_{c}\right.$ and $\left.R_{N}\right)$ to achieve a non-hysteretic regime.

Acknowledgements We thank Manuela Popescu and Martijn Schoemans for their precious technical help.

Open Access This article is distributed under the terms of the Creative Commons Attribution Noncommercial License which permits any noncommercial use, distribution, and reproduction in any medium, provided the original author(s) and source are credited.

\section{References}

1. L. Gottardi et al., in Proceedings of the Low Temperature Detectors Conference, vol. 13, ed. by B. Cabrera, A. Miller, B. Young (2009), pp. 245-248

2. L. Gottardi et al., in Proceedings ASC (2011)

3. N. Iyomoto et al., Appl. Phys. Lett. 92, 13508 (2008)

4. S.R. Bandler et al., J. Low Temp. Phys. 151, 400 (2008)

5. J. Sadlier et al., Phys. Rev. Lett. 104, 047003 (2010)

6. L.D. Jackel, R.A. Buhrman et al., J. Low Temp, Physics 19, 3-4 (1975)

7. J. Kurkijärvi, Phys. Rev. B 6, 832 (1972)

8. J. Kurkijärvi, J. Appl. Phys. 44, 3729 (1973)

9. M.B. Simmonds, W.H. Parker, J. Appl. Phys. 42, 38 (1971)

10. A.H. Silver, J.E. Zimmerman, Phys. Rev. 157, 317 (1966)

11. J. Clarke, A.L. Braginski, The SQUID Handbook (Wiley, New York, 2004)

12. S.J. Smith et al., Submitted to J. Appl. Phys. (2011) 